\title{
Perspectiva de la enfermera ante el paciente oncológico en fase terminal en Tampico, Tamaulipas, México
}

\author{
Perspective from the nurse to the terminal cancer patient in \\ Tampico, Tamaulipas, Mexico
}

\section{Perspectiva do enfermeiro ao paciente com câncer terminal em Tampico, Tamaulipas, México}

Dra. María Elena Pérez Vega ${ }^{1}$; Dr. Luis Cibanal Juan ${ }^{2}$

${ }^{1}$ Facultad de Enfermería, Universidad Autónoma de Tamaulipas, México

${ }^{2}$ Departamento de Enfermería, Universidad de Alicante

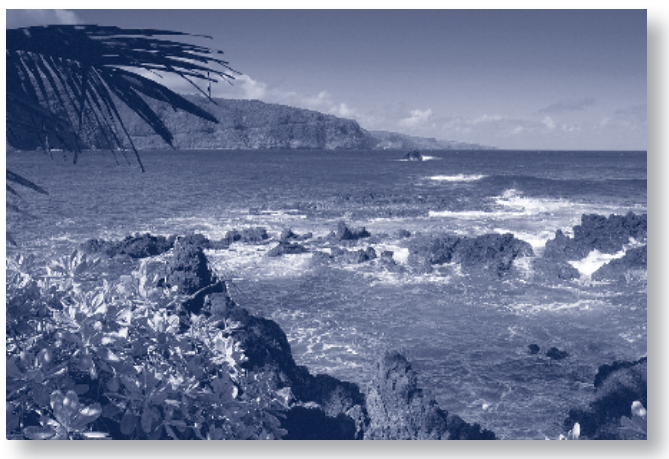

Perspective from the nurse to the terminal cancer patient in Tampico, Tamaulipas, Mexico

\section{ABSTRACT}

This paper presents the results of a qualitative study conducted in three hospitals in Tampico, Mexico. It details a different perspective from the last stage of human life, specifically, the work of the nurse in the care of terminal cancer patient, and her relationship with him and his families when he dies.

Within nursing practice, it must be given special emphasis to the care of terminally ill patients. During this phase, the patient experience different symptoms, which are complex and changing, therefore he needs to have a holistic and ethical care to improve their quality of life wherever possible.

However, the results of this inquiry showed that nurses experience the process of dying with pain, sadness and powerlessness. The pain and sadness are associated with the importance that nurses give the patient, also by their mutual relations. They experience impotence, feeling they could not do more for him, and believe that the effort, time and resources devoted for his attention were vain.

Keywords: Terminal cancer patient, terminally ill patient, terminal phase, death.

Perspectiva do enfermeiro ao paciente com câncer terminal em Tampico, Tamaulipas, México

\section{RESUMO}

Este artigo apresenta os resultados de uma pesquisa qualitativa foi realizada em três hospitais de Tampico, Tamaulipas, México. Ele detalha uma perspectiva diferente da última etapa da vida humana. Especificamente, o trabalho do enfermeiro no cuidado de 
pacientes terminais de câncer e sua relação com ele e sua família quando o tempo está se aproximando da morte. Dentro da prática de enfermagem não é uma questão que deve ser dada ênfase especial: o cuidado de doentes terminais. Durante esta fase, o paciente experimenta sintomas diferentes, que são complexas e em mudança, então você precisa de uma atenção integral e ética para melhorar sua qualidade de vida sempre que possível. No entanto, os resultados desta pesquisa mostram que as enfermeiras vivem o processo de morrer de tristeza, dor e desamparo. A dor ea tristeza que lhes estão associados significa que o paciente eo tempo de convivência mútua. A sensação de impotência experiência que poderia fazer mais por ele ou não serve muito de todos os tempo, esforço e recursos destinados a sua atenção.

Palavras-chave: doentes com cancro, doentes terminais, doentes terminais, a morte.

\section{RESUMEN}

En este trabajo se muestran los resultados de una investigación cualitativa que se realizó en tres hospitales de Tampico, Tamaulipas, en México. En ella se detalla una perspectiva distinta respecto a la última etapa de la vida humana. Específicamente, la labor de la enfermera en el cuidado del paciente oncológico terminal y su relación con él y su familia cuando se acerca el momento de la muerte.

Dentro de la práctica de enfermería existe un asunto al que se le debe dar un énfasis especial: el cuidado de pacientes con enfermedades terminales. Durante esta fase, el enfermo experimenta diversos síntomas, que son complejos y cambiantes, por ello necesita tener una atención integral y ética para mejorar su calidad de vida dentro de lo posible.
No obstante, los resultados de esta investigación mostraron que las enfermeras viven el proceso de muerte con dolor, tristeza e impotencia. El dolor y la tristeza se relacionan con lo que les significa el paciente y por el tiempo de convivencia mutua. La impotencia la experimentan al sentir que no pudieron hacer más por él o que no sirvieron de mucho todo el esfuerzo, tiempo y recursos dedicados a su atención.

Palabras Clave: Paciente oncológico, enfermo terminal, fase terminal, muerte.

\section{CONSIDERACIONES TEÓRICAS}

Por las responsabilidades inherentes a su profesión, la enfermera continuamente enfrenta la realidad de la muerte de otras personas. Este acercamiento genera emociones y sentimientos de difícil manejo: confusión, dolor, pérdida de esperanza, miedo, rabia, agotamiento, soledad, ambivalencia, necesidad de escapar, sensación de ser intrusos, deseos de evitar al enfermo y a la familia, fuertes deseos de que el enfermo se alivie y viva, junto con deseos igualmente fuertes de que ya muera (Reyes, 1996). La situación del paciente que atraviesa una agonía invadida de dolor, tanto emocional como físico, puede despertar en ella inquietudes, ansiedades e impotencia, ya que por un lado, de forma inconsciente, le supone enfrentarse a su propia muerte, aumentando sus temores personales sobre ésta y por el otro, el aceptar la muerte del paciente contradice el objetivo esencial de la atención sanitaria, que es conservar la salud y la vida.

Yague y García (1994) señalan que la presencia de la muerte remueve también las actitudes del personal de enfermería y, recíprocamente, esas actitudes influyen positiva o negativamente en el proceso terminal del paciente al que se está asistiendo. Cuando un in- 
dividuo entra en un proceso terminal se convierte en un estímulo que despierta aversión y genera respuestas de evitación y huida. $Y$ aún cuando el enfermo encuentre gran alivio y se sienta confortado cuando puede hablar de sus sentimientos y compartir sus preocupaciones respecto a la muerte, el profesional de enfermería puede manifestar las siguientes actitudes: a) tendencia a evitar que el enfermo se entere de cuál es su verdadero estado, o posponer al máximo esta información, b) apartarse física o emocionalmente del moribundo, para evitar pensar en la propia muerte y sentir miedo, y c) tendencia a extremar las medidas terapéuticas tratando de alcanzar la curación del paciente, consiguiendo que se disminuya la calidad de vida y se alargue la agonía.

Como vemos, no sólo se le teme a la muerte, sino que se le rechaza y niega. Estos cambios actitudinales también han alcanzado a los profesionales de la salud, generando muchas veces que no quieran nombrar a la muerte $o$ a las patologías "que la atraen" o no mirar cara a cara al enfermo terminal, (Gala, Lupiani y Díaz, 1991) y el aumento de la atención tecnológica en detrimento de la empático-afectiva, con el riesgo del encarnizamiento terapéutico, empeorándose las condiciones de la muerte. Por consiguiente, una enfermera puede sentir como algo "positivo" la atención al enfermo en fase terminal, pero no necesariamente actuar de acuerdo a ese sentimiento.

La atención de los pacientes oncológicos en fase terminal en los hospitales de Tampico, Tamaulipas: un contexto.

Tampico es una importante ciudad y puerto enclavada en el sudeste del estado de Tamaulipas, en México. Es la zona metropolitana costera con mayor población en el Golfo de México y la segunda en importancia econó- mica en la República Mexicana. Se caracteriza por estar dedicada casi exclusivamente al comercio y es cuna de dos consorcios con gran escala en el noreste del país.

En cuanto al sector salud, éste representa una significativa fuente de empleos e ingresos en la zona. Se conforma por más de doce hospitales públicos y privados y un cuerpo médico compuesto por más de 1200 médicos que ejercen entre más de 45 especialidades de medicina. Además, se tienen al alcance varias clínicas especializadas, laboratorios, y gabinetes de diagnósticos con lo más avanzado en tecnología y tratamientos médicos. La oferta médica en Tampico, es la más importante de la región.

En Tampico los pacientes oncológicos reciben tratamiento ambulatorio, es decir, se programa su sesión de quimioterapia y el paciente acude el día señalado; en seguida de recibir la sesión se va a su casa. Generalmente son atendidos en el área de quimioterapia por enfermeras que en su mayoría no tienen la especialidad de Oncología, sino que son intensivistas, licenciadas o enfermeras generales; por lo tanto, es posible que no tengan la intervención adecuada para el paciente, ya que requieren poseer no solamente conocimientos sobre terapéuticas para el control sintomático, sino también conocer y detectar las manifestaciones y necesidades por las que transita el paciente oncológico terminal (emocionales, espirituales y físicas) para poder brindar una atención activa, global y holista. Así, se puede suponer que con el paciente oncológico terminal se produce el denominado "triangulo del sufrimiento", donde no sólo sufre el paciente, sino también la familia y el equipo de salud (Loncán et al., 2007).

Hemos visto que la muerte en el medio hospitalario no se ve como un proceso natural. En los hospitales de Tampico, Tamaulipas, el 
paciente en fase terminal generalmente se encuentra en el cuarto más alejado a la Central de Enfermeras y casi siempre está solo; o bien, puede estar en uno de los cuartos cercanos a la Central de Enfermeras con otro paciente que esté en condiciones similares, pero de igual manera solo.

\section{ASPECTOS METODOLÓGICOS}

Para llevar a cabo esta investigación se diseñaron y aplicaron a cada informante dos instrumentos de recogida de información: una cédula de datos sociodemográficos y una guía de entrevista semiestructurada. Con la cédula sociodemográfica personal se obtuvieron datos acerca de la institución y servicio de hospitalización, turno asignado, nivel académico, edad, antigüedad laboral, antigüedad en el servicio de quimioterapia/hospice, realización de cursos sobre pacientes oncológicos y religión.

Por su parte, los ítems de la guía de entrevista fueron: concepto y significación de la muerte, significación de la propia muerte, significación del paciente en fase terminal, necesidades del paciente oncológico en fase terminal, contexto hospitalario de atención al paciente, competencias para el manejo de estos pacientes, quehacer de la enfermera, impacto emocional en la enfermera, acompañamiento de la familia, experiencia vivida ante el primer paciente atendido.

Las seleccionadas fueron diez licenciadas en enfermería de tres hospitales de segundo nivel de atención de la ciudad y puerto de Tampico, Tamaulipas, México. De las diez informantes, dos cumplen el rol de docentes y siete tienen el referente de haber vivenciado personalmente la muerte de un ser querido o cercano; dos informantes tienen la especialidad de Oncología y una declaró no haber realizado cursos sobre cuidados paliativos o pacientes terminales. La edad promedio de las entrevistadas fue de 40 años, siendo la mínima de 25 años y la máxima de 55 años cumplidos. Todas las informantes profesan la religión católica.

Para el análisis de la información recabada, se emplearon diez documentos estenográficos, resultado de la trascripción de las audiograbaciones de entrevistas semiestructuradas. El procesamiento de la información fue de tipo manual al estilo tradicional antropológico.

\section{RESULTADOS DE LA INVESTIGACIÓN}

Con base en las narrativas analizadas, hemos encontrado que existe una fuerte confrontación emocional en las enfermeras, entre lo que sienten y lo que les han enseñado que son los límites y el distanciamiento emotivo deseable. Hay una idea personal, hospitalaria y de formación profesional de que la enfermera debe ser un ser escindido entre su yo personal y su yo profesional, que contradictoriamente rechazan y aceptan en su discurso, lo que les origina una angustia existencial profunda, un torrente de emociones contenidas y un caos en los parámetros para el comportamiento cotidiano.

El enfermo terminal es visto por parte de las entrevistadas como alguien con padecimientos y cuya enfermedad tan avanzada no le permite la pronta respuesta a tratamientos, por tanto sus síntomas, además de físicos cambiantes, también son emocionales e intensos. El enfermo terminal es alguien que requiere del apoyo no sólo especializado (de ellas), sino de la familia, pero también de un entorno o contexto hospitalario sensible e integral. La actuación de la enfermera, entonces, sí es medular, pero no independiente de la actuación de otros sujetos que deben o están implicados de forma natural, pues aunque ellas están direccionadas a la asistencia y acompañamiento del paciente, las experiencias humanas que 
éste proceso conlleva, obliga a "socializar" esta actuación; es decir, compartirla a través del discurso generado desde o hacia los miedos, la preocupación, el desdén, la desesperación o la paciencia de quienes rodean al paciente e, incluso, de quien ellos se hacen rodear: Dios, por ejemplo.

Si evaluáramos el nivel de conocimientos que posee la enfermera sobre los cuidados paliativos, sugeriríamos que éste se encuentra en un punto básico, es decir, limitado a cuidados físicos: aliviar el dolor, aunque sí se mencionen otros factores determinantes, el ayudar a la familia a afrontar tal situación es un momento posterior o secundario. Una riqueza importante en este estudio radica en que la enfermera está consciente de la importancia de la actualización constante a través de cursos para su formación no sólo disciplinaria, sino emocional.

Sobre la idea inicial de este estudio de una vivencia en soledad del proceso terminal y la muerte en los pacientes, el discurso de las informantes no muestra elementos para sostener tal planteamiento. Por el contrario, hay evidencia en el texto para afirmar que quien vive en soledad el proceso de la fase terminal es la enfermera, pues para ella no se refiere ningún acompañamiento de apoyo y sí un fuerte impacto emocional. Los profesionales sufren en solitario la batalla entre la vida y la muerte, evidenciándose que necesitan soporte emocional para experimentar el luto.

Por otra parte, fue notorio que la experiencia de tratar a estos pacientes y ver el deterioro progresivo que van presentando, así como la dinámica familiar que los rodea, sí afecta a las enfermeras, pues por lo menos dos de ellas (una del hospice y otra del servicio de quimioterapia) lloraron al recordarlos, que fue cuando se les pidió que hablaran del paciente que les haya sido más significativo.
En otro tenor, es notorio que se continua dando prioridad al tratamiento de los síntomas que el paciente refiere, siendo el dolor el más importante, quizá porque el contacto cercano con el deterioro progresivo que el paciente presenta las va dejando vulnerables ante una escena que la mayor parte de las veces no se quiere presenciar.

Se evidencia en este estudio que muchas actitudes de distanciamiento de la enfermera ante el paciente en fase terminal pueden deberse a: la respuesta personal de acatamiento al supuesto deber ser profesional, que plantea el control de sentimientos y la no vinculación emocional; a la carga de trabajo y el poco tiempo disponible; pero sobre todo, a mecanismos psico-afectivos de defensa ante los sentimientos de un duelo prolongado y permanente o a efectos patológicos de este duelo profesional cotidiano, como sería la depresión..

La enfermera ayuda a bien morir, entendiendo por esto, el morir con paz espiritual. El paciente en fase terminal y su familia llegan a la paz espiritual gracias a la presencia y actuación de la enfermera, quien vincula al paciente con Dios y contrarresta hasta cierto punto, el contexto adverso hospitalario.

Por otra parte, ante la muerte próxima del paciente y la reflexión sobre la muerte misma, la enfermera busca su propia paz espiritual (emocional), la cual nunca es alcanzada debido a su rol profesional y a su afrontamiento continuo con la fase terminal. Su falta de paz se agrava si el paciente no logra la paz espiritual por la no aceptación de él mismo o de su familia, por el contexto hospitalario adverso a bien morir o la incompetencia profesional de ella misma como enfermera. Las enfermeras también consideran que su preparación básica en enfermería es inadecuada para proporcionar cuidados al paciente en fase terminal. 


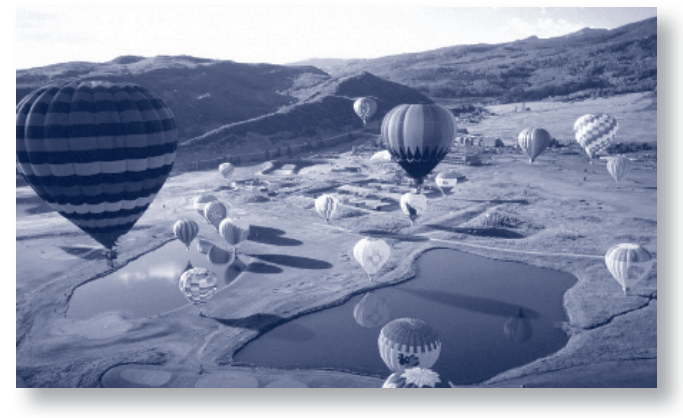

Ante el conocimiento de los recursos para abordar la enfermedad, podemos decir que la competencia o capacidad profesional, se entiende como la posibilidad de hacer lo debido, sin embargo, en el concepto expresado en la narrativa sobre el deber ser, se evidencia una fuerte noción de ambigüedad y contradicción que pone en confrontación permanente polos de acción y valor: sentir y no sentir, priorizar la atención corporal, para lo que la enfermera se siente profesionalmente capacitada, o priorizar la atención espiritual, que en lo personal considera el deber ser humanitario de la enfermera y de ella como ser humano. Frecuentemente la enfermera, según su narración, prioriza lo espiritual, pero se asume por ello como irreverente a su aprendizaje escolar. Además, para atender lo espiritual no posee formación ni recursos como enfermera, sino sólo los que sus experiencias y vivencias personales le proveen.

El dolor y el sufrimiento también constituye un eje de contradicción narrativa, ya que se ve, por una parte, como algo negativo a evitar y que constituye el foco central de la idea de "bien morir" y de paz emocional, mientras que, por otra parte, se le ve como una fuerza positiva que purifica al paciente en fase terminal y le permite conseguir la paz espiritual y llegar a Dios.

La enfermera establece una relación de contacto físico y emocional con el paciente en fase terminal. Esta cercanía le provoca sentimientos fuertemente dolorosos que la impactan emocionalmente, ya que transita por due- los permanentes y sucesivos por el complejo e intenso manejo de los aspectos humanos y la vivencia del proceso de morir.

El proceso de agonía en solitario no es vivido por el paciente terminal sino por la enfermera, quien enfrenta el paradigma profesional entre el sentir y el no deber o no querer sentir. Por supuesto, lo lógico y lo humano es que lo sienta, y esto por empatía, pero que también debe no confundir empatía con simpatía, donde en este caso se mete en el sufrimiento del otro sin salir de él, lo que ya no es empatía; con el consiguiente peligro de que se queme. La enfermera, si ha habido relación con el paciente, lógicamente tendrá que hacer también el duelo al morir éste, esto es lo humano; pero claro, su duelo no es lo mismo que el de la familia, pues no forma parte de la familia del paciente.

Por otra parte, al identificar el papel que desempeña la familia en el proceso terminal del paciente oncológico, se reflexionó que ésta, más allá de ser una estructura conformada desde una visión clásica como un órgano de parentesco y dependencia que actúa como agente socializador; conjuga más bien cierto sentido de existencia entre sus integrantes, que brinda un significado de vida, sea éste negativo o positivo. Así, al interior de las familias pueden encontrarse miembros que se interesan en brindar cuidado y protección al familiar en fase terminal; pero desafortunadamente esto no sucede con regularidad. La enfermera observa entonces, que el mayor reto al que se enfrenta el paciente oncológico en fase última es a la incomprensión y ésta, indudablemente, se halla en la mayoría de los casos en el seno de la familia, pues implica llegar a un nivel superior de relación con el otro, por el otro y para el otro.

En la perspectiva menos favorable, la familia también puede convertirse en un obstaculizador para la comprensión de la enferme- 
dad ya que al ser un sistema, tiene relaciones económicas, sociales y políticas que la hacen estar vulnerable al contexto y al ambiente, de tal manera que más allá de convertirse en una responsabilidad ética, se convierte en una obligación, que no siempre es amable ante carencias, limitaciones y salvedades muchas. En lo ideal la familia, desde los discursos analizados, debiera ser un punto de encuentro, de corresponsabilidad, de colaboración y de confianza.

Finalmente, observamos que la relación de todos los actores involucrados en el proceso de confrontación y aceptación de la muerte se define a través de dos dimensiones, una reduccionista y la otra con paradojas y conflictos de identidad. La primera, remite a que el hombre es sólo una entidad física, sin distinción de otro de su misma especie; lo que hace que lenguajes, relaciones y tratamientos se unifiquen hacia una sola dirección, desconociendo que el paciente y su enfermedad no son sólo producto de un "problema de su organismo", sino de un sistema de eventos que tienen que ver con sus formas de vida económica, anímica, existencial, social e incluso política.

La segunda implica que, ante el rechazo de las narrativas espirituales frente a la rígida doctrina científica en la que ha crecido la medicina, la aceptación de que sólo se cumple un papel y la negación a observarnos como sujetos morales y con fe, se genera un conjunto de contradicciones que obligan, sobre todo a los profesionales de la salud, a decidir si siguen lo que debe ser (socialmente sancionado y aceptado históricamente) o asumen su condición humana para las relaciones con los pacientes.

\section{Propuesta de mejora}

A raíz de esta investigación, advertimos necesarias las siguientes acciones urgentes:
- Reorientar la atención hospitalaria del paciente en fase terminal bajo criterios tanatológicos. Esto es, la muerte como proceso multidimensional por su carácter biológico, psicológico, social y religioso o espiritual, requiere ser abordado por un equipo multidisciplinario capacitado para disminuir el sufrimiento físico y psicológico por el que está transitando el paciente, apoyar a la familia para reducir la aprehensión y los sentimientos de culpabilidad y pérdida y evitar la frustración y favorecer el manejo de los duelos en el equipo de salud; especialmente en la enfermera, quien no escapa fácilmente del conflicto generado por la muerte y la agonía.

- Preparar al personal de enfermería para:

- Enfrentar una pérdida, manejar una enfermedad

- Apoyar al familiar y al paciente en el transcurso de la enfermedad terminal

- Manejar su duelo personal de manera que no genere bloqueos emocionales que se reflejen en tensiones corporales o enfermedades.

- Romper con el paradigma profesional que confronta a la enfermera entre el sentir y el no deber o no querer sentir y

- Brindar apoyo especializado al personal de enfermería, como una medida urgente de salud mental laboral, ya que el riesgo de depresión y afectación del Síndrome de Burnout es muy alto.

\section{CONCLUSIONES}

Con base en el estudio realizado en Tampico, México, se tuvo la oportunidad de identificar algunos aspectos de gran interés en el área de atención a enfermos terminales. Principalmente, se identificaron, al interior de los hospitales, algunas carencias en este tipo de servicios. 
Primeramente, se observó que es necesario orientar el concepto y la práctica médica en su enfoque perceptivo, para que tanto institución como actores estén involucrados en el mismo sentido denominador dentro de la actividad de preservar la vida y comprender la muerte, a través de la creación de algo más que un lugar donde los padecimientos se vean como orgánicos, con un ambiente y un escenario en el que los desequilibrios de los seres humanos son el producto de múltiples factores que definen y orientan la situación de salud de las personas, no sólo del paciente, sino de los demás, lo que implicará que en lugar de ser centros hospitalarios, se vuelvan comunidades de salud integral.

De hecho, es preciso que el médico se vea como paciente y/o familiar, que el mismo paciente se vea como "cuidador" o familiar, la enfermera como sanadora y paciente, la familia como paciente o institución médica, para que a través de este punto de vista se asuma que alguien puede vivir esas situaciones y con ello entender al otro de esa manera y atenderlo en consecuencia, en definitiva esto se traduciría en una empatía continua ante cualquier situación de las que estamos señalando. Para ello, se requiere integrar a la dinámica de esos lugares integrales de bienestar, los discursos religiosos y emocionales, no como terapias alternativas, sino como elementos que posibilitan un sentimiento de pertenencia a un mundo que es espiritual e inyectar a estos lugares de ese halo de "misterio" que es necesario en los momentos de alivio añorado. Se trata de "entrar" en la comprensión de la realidad del paciente terminal y de los demás sujetos.

Finalmente, el cuidado enfermero no es algo acabado. En concreto, lo que siempre debe estar en proceso de conformación es un alto sentido de comunicación humanista en el que dominen: la tolerancia, la empatía, el fee-

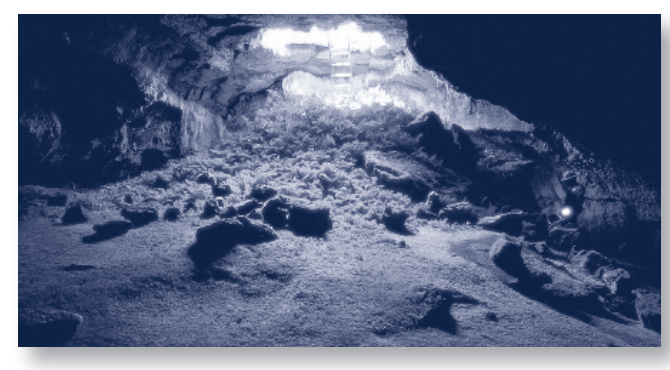

dback, la paciencia, el entusiasmo, la reciprocidad, la responsabilidad, la ética, la comprensión e, incluso, el sentido de ciudadanía. La existencia es el valor más preciado de todos los seres humanos; por eso, tanto médicos como enfermeras deben esforzarse por brindar los mejores cuidados a los pacientes terminales, y un servicio óptimo a sus familiares. El mundo de la enfermería puede ser uno de los mayores ejes para lograr un vivir mejor. No se trata de palabras sordas, es más bien un principio esperanzador.

\section{REFERENCIAS}

- Bregel, F., (2004) "Hablando de la muerte en el aula" [En línea], disponible en: http://www.metas.org/educare/ educare11/ensenando/ensenando1.pdf

- Gala, J., Lupiani, M. y M. Díaz, (1991) Sobre las concepciones de la muerte. Rev. Rol., 159: 63-66.

- Loncán, P., Gisbert, A., Fernández, C. et al. (2007) Cuidados paliativos y medicina

- Intensiva en la atención al final de la vida del siglo XXI. Anales Sis San Navarra [En línea], disponible en: http:// www.cfnavarra.es/salud/anales/textos/vol30/sup3/suple9.html

- Reyes, L., (1996). Curso Fundamental de Tanatología. Acercamientos tanatológicos al enfermo terminal y a su familia. México, Edición de autor.

- Scott, D., (1988). Is paliative care a discipline? Palliat Care, $4: 10-11$

- Yague, A. y García, M., (1994) “Actitudes de los profesionales de enfermería ante los pacientes terminales" [En línea], disponible en: http://www.uclm.es/ab/enfermeria/ revista/numero\%204/terminales4.htm 